\title{
DIE VERGESSENE ROLLE VON JOHANN IGNAZ VON FELBIGER IN UNGARN
}

\author{
EVA KOWALSKÁ \\ Historisches Institut der Slowakischen Akademie der Wissenschaften ${ }^{1}$ \\ eva.kowalska@savba.sk
}

\begin{abstract}
Der Beitrag widmet sich der Schulreformen in Ungarn und im Besonderen der Rolle von Johann Ignaz von Felbiger (1724-1788). Seine Pressburger Jahre (17791788) werden kommentiert, jedoch nur in Anfangsphase seiner Tätigkeit in Ungarn. Es wird gerne behauptet, dass er dorthin eigentlich verbannt worden sei. Die neuere Forschung, so auch diese Studie, erhellt die frühere Rolle von Felbiger in österreichischen und tschechischen Ländern, die eine ganz ähnliche war: Er sollte Anfang der 1780er Jahre das System der Trivialschulen in Ungarn in Schwung bringen und seine Kompatibilität mit jenem in hereditären Ländern gewährleisten. Der Verlust seiner Position hing mit dem Wechsel in der Schulpolitik nach 1781 zusammen.
\end{abstract}

Schlüsselwörter: Johann Ignaz von Felbiger; Ungarn; Schulwesen; Reformen; Aufklärung; Maria Theresia; Joseph II.

Die die Schulreformen in Ungarn betreffende ältere sowie neuere Literatur registriert zwar die Aufgabe von Johann Ignaz von Felbiger bei der Gestaltung des staatlichen Elementarschulwesens, aber sie konzentriert sich nur auf die Anfangsetappe seiner Tätigkeit in Ungarn. ${ }^{2}$ In den Kommentaren seiner letzten Jahre, die er in Pressburg verbrachte (1779-1788), wird allgemein behauptet, dass er eigentlich dorthin abgeschoben wurde, und zwar wegen seiner Kontroversen mit Joseph II. bezüglich des Elementarschulwesens, insbesondere bezüglich der Elementarschulen für Soldatenkinder. ${ }^{3}$ Die bisher nicht berücksichtigten, obwohl bereits publizierten Archivdokumente, die das Slowakische Nationalarchiv aufbewahrt, ${ }^{4}$ ermöglichen jedoch eine ausführlichere und dieser These widersprechende Interpretation. Sie verweisen darauf, dass Felbiger direkt im Auftrag Maria Theresias und später auch Josephs II. in Ungarn eine ähnliche Rolle spielen sollte, wie er sie in Böhmen und den österreichischen Erbländern innehatte: In seiner Wirkungsstätte in Pressburg sollte er sich an der Vereinheitlichung des Systems der Volksschulbildung im Rahmen der gesamten Monarchie beteiligen. Umso mehr, dass der Reformplan Ratio educationis im Kapitel über die Volksschulen jenes Modell kopierte, das Felbiger in den Erbländern einführte. Nur hier, in der Hauptstadt Ungarns, konnte er einen unmittelbaren Kontakt zum ungarischen Milieu gewinnen, wobei die Zuerkennung eines bedeutenden Kirchenran- 
ges Garantie für sein Ansehen und seine Akzeptanz sein sollte. ${ }^{5}$ Wahrscheinlich rechnete man mit Felbiger auch bei den beabsichtigten Verhandlungen mit den ungarischen Protestanten hinsichtlich der Unterordnung ihres Schulwesens unter die staatliche Verwaltung, da seine Kontakte und Bewunderung des deutschen protestantischen Schulwesens sowie die gemäßigte tolerante Haltung allgemein bekannt war. ${ }^{6}$ Der allmähliche Einflussverlust in seinen letzten Lebensjahren hing mit dem Eintritt der neuen Generation von Reformatoren um Gottfried van Swieten zusammen, die zu einem gewissen Grad jenes Modell der Normalschule verachteten, das Felbiger bereits entwickelt hatte.

Seine Ernennung zum Propst von Pressburg (am 22. Januar 1779) brachte Felbiger in jene Stadt, die er bereits ein Jahr nach seiner Ankunft in Wien, vom 3. bis 8. April 1775 besucht hatte. Er hatte direkten Anteil an der Bestellung der Volksschulkommission beim Ungarischen Königlichen Statthaltereirat und auch an der Eröffnung der ersten Normalschule Ungarns in Pressburg, am 30. Juni 1775. ${ }^{7}$ Die ungarische Öffentlichkeit wurde über seine Aktivitäten aber schon früher in der Preßburger Zeitung informiert ${ }^{8}$ und es stand ihr auch ein lateinischer Auszug aus seinen Werken, betreffend seine Auffassung von Elementarlehre und Unterrichtsmethoden, zur Verfügung. ${ }^{9}$ Sogar sein Katechismus wurde noch vor seiner Genehmigung durch die österreichischen, geschweige denn der ungarischen Bischöfe, auf den kaiserlichen Güttern Holitsch und Sassin eingeführt. ${ }^{10}$ Bereits in der Zeit der Vorbereitung der Allgemeinen Schulordnung beteiligte sich Felbiger dann auch persönlich, noch in Wien verweilend, am Geschehen im ungarischen Schulwesen: Seit Anfang 1774 erwog man die Eröffnung einer Normalschule in Pressburg und am 18. März 1774 schlug man einen Ex-Jesuiten, Pater Otto, als Direktor dieser Schule vor und empfahl ihn mit 2, 3 anderen Lehrern nach Wien zu Felbigers Lehrerkurs. ${ }^{11}$ Als Otto wahrscheinlich wegen seiner Ordensvergangenheit abgelehnt wurde, rechnete man seit Mai 1774 mit Martin Hoffmann, der bereits bei Felbiger in Wien einen Kurs absolvierte. Hier griff aber wieder Felbiger selbst ein, der statt Otto Johan Roka (den Autor des erwähnten lateinischen Reformplanes Institutiones) protegierte, der das Ernennungsdekret auch am 25. November 1774 erhielt. ${ }^{12}$

Der erste Besuch von Felbiger in Pressburg von 3. bis 8. April 1775 beendete seine zuvor nur indirekten Kontakte mit Ungarn. Bis dahin führte Felbiger die Tagungen über die Bildung einer Kommission für Volkschulen beim Statthaltereirat, wobei sie zur Zweigstelle oder sogar einem Bestandteil der Studienhofkommission werden sollte. ${ }^{13}$ Die explizite Aufgabe der Kommission bestand darin, die Normalschulen und die Grundsätze der Allgemeinen Schulordnung ins Leben zu rufen, wobei sie sich am Entwurf der Instrukzion für die Schulkommission der Erbländer, die am 28. Dezember 1774 erlassen worden war, halten sollte. ${ }^{14}$ Auf diesem Entwurf basierten auch die Bestimmungen für einen Inspektor der Elementarschulen in Ungarn. Alle diese Indizien weisen darauf hin, dass man in 
den Jahren 1774-1775 noch keine selbstständige Reform - zumindest nicht für das Elementarschulwesen - in Ungarn erwog.

Die Elementarschulkommission entstand einen Monat nach Felbigers Aufenthalt in Pressburg. Ihre Mitglieder waren Franz Balassa als Präses, der Neusohler Bischof Franz Berchtold, der Statthaltereirat Nicolaus Skerlec, Johann Zgurics und später auch Volksschulinspektor Caspar Pál ab Ehrenfels als ordentliche Mitglieder. Die erste Aufgabe der Kommission war die Einrichtung der Normalschule in Pressburg, was sich abgesehen von den relativ guten Raum- und Finanzbedingungen als eine ganz schwierige Sache erwies. ${ }^{15}$ Man musste langwierige Verhandlungen mit dem Magistrat führen, um seine Unterstützung zu gewinnen und außer der Anpassung der Räumlichkeiten im ehemaligen Jesuitenseminarium auch die benötigten Bücher besorgen zu können. ${ }^{16}$ Trotz dieser Probleme gelang es der Kommission, die Pressburger Normalschule bereits am 30. Juni 1775 zu eröffnen. Die Bedeutung dieses Schritts wird um so größer, wenn man ihn mit den entsprechenden Entwicklungen im Nachbarland Böhmen vergleicht: Zu dieser Zeit galt dort zwar die Allgemeine Schulordnung bereits als Norm, doch trotzdem begann man erst mit den Vorbereitungen zur Eröffnung einer Normalschule in Prag. Obwohl in Böhmen bereits früher auf den großen Grundherrschaften Reformversuche unternommen wurden, ${ }^{17}$ verschob sich durch die Absenz eines zentralen Koordinationszentrums der Gründungstag der Normalschule in Prag erst auf den 15. November 1775. Daraus ergibt sich die Frage, ob diese Verschiebung nicht als Beweis für die symbolische Betonung des angestrebten Vereinheitlichungstrends in Rahmen des Schulwesens zu verstehen ist...

Es gibt keinen direkten dafür, dass Felbiger bereits nach 1775 engere Kontakte mit den leitenden Schulwesensbehörden in Ungarn pflegte und sich - in Wien wirkend - an der Versorgung der Pressburger Normalschule mit Lehrbüchern beteiligte. Wegen der Probleme mit dem Bücherimport war doch die Studienhofkommission gezwungen, sich am 3. Juni 1775 mit Felbigers Vorschlag zu befassen und die Privilegien für den Lehrbuchdruck auch den privaten Druckereien, und zwar auch außerhalb der österreichischen Länder, zu erteilen. ${ }^{18}$ Dieses Modell wurde tatsächlich umgesetzt - in Ungarn erhielt ein solches Privileg zuerst Katarina Landerer in Ofen (Buda), später Johann Michael Landerer und Augustin Franz Patzko in Pressburg, und erst 1779 ging das Privileg des Lehrbuchdrucks definitiv auf die Universitätsdruckerei über. ${ }^{19}$

Mit der Eröffnung der Normalschule in Pressburg endete in Ungarn jedoch vorläufig jene Etappe, in der man an die Schaffung eines einheitlichen Systems des Volkschulwesens innerhalb der gesamten Monarchie dachte. Doch in den obersten Regierungskreisen in Wien begann eine Diskussion über die Selbstständigkeit Ungarns auch im Bereich des Schulwesens und allmählich setzte sich die Idee einer separaten und komplexen Reform für Ungarn durch (CsóKA, 1939). ${ }^{20}$ Erst in dieser Phase gewann in Ungarn Adam Franz Kollar, der kurz zuvor mit 
einem Reformplan für Gymnasien in den Erbländern beauftragt worden war, aber in seinen Bemühungen daran scheiterte, an Bedeutung. Ein gemeinsames Projekt mit Daniel Terstyánszky und Joseph Ürményi Ratio educationis legte den Grundstein für ein selbstständiges ungarisches Schulsystem, auf dem Gebiet des Elementarschulwesens blieb man jedoch den bisher bekannten und applizierten Prinzipien und de facto Felbigers Allgemeiner Schulordnung (betreff. Unterrichtsmethode, Schultypen) treu: Der Unterrichtsplan der Pressburger Normalschule kopierte das Wiener Vorbild, man importierte und benutzte Felbigers Lehrbücher, nach 1778 begann man sogar allmählich mit ihrer Übersetzung in Nationalsprachen. ${ }^{21}$ Es wurde auch die Kernforderung Felbigers, d.h. die Institutionalisierung der Lehrerausbildung in Form der Präparandie umgesetzt. Die Zahl der so reformierten Schulen wuchs jedoch nur langsam und diese Situation entsprach nicht den Erwartungen der Reformatoren.

Wie bereits erwähnt, wurde mit dem Erlass von Ratio educationis (1777) die organisatorische Initiative völlig den ungarischen Verwaltungsorganen übertragen und Felbigers unmittelbarer Einfluss ist in dieser Periode nicht belegt. Er erhielt nur die Beauftragung zur „Reformation“ des Unterrichts Preßburger Mädchenpensionat Notre Dame, der Lieblingsinstitution Maria Theresias, der sie auch selbst Besuche abstattete. Eine Wende trat ein, als sich nur geringe Fortschritte bei der Einführung der ,,verbesserten“ Schulen, die zwei Jahre seit der Einführung von Ratio educationis nur in einigen größeren Städten errichtet wurden, bemerkbar machten. Der Präses der ungarischen Volksschulkommission Franz Balassa arbeitete zwar fleißig, aber sein Hauptinteresse richtete sich vor allem auf die hohe Politik. ${ }^{22}$ Ein anderes Kommissionsmitglied, der Neusohler Bischof Berchtold, musste vor allem die Verhältnisse in seinem neugegründeten Bistum organisieren. Die Volkschulinspektoren waren mit Arbeit überhäuft: neben den Bemühungen um Einführung des neuen Unterrichts mussten sie auch Übersetzungen der Felbigerschen Lehrbücher vorbereiten (in Böhmen war dafür ein Verlag für die Normalschule in Prag, ähnlich wie in Wien, verantwortlich). Wahrscheinlich noch zu Lebzeiten von Maria Theresia wurde die Anstellung ,neuer Leute“ erwogen, die fähig wären, vor allem das System der Lehrerausbildung und der Herausgabe von Lehrbüchern in Gang zu bringen, den Inhalt des Unterrichts an die Verhältnisse in Ungarn anzupassen und die Stadtmagistrate und Stadtobrigkeiten dazu zu bewegen, die Verantwortung für den Schulbetrieb zu übernehmen. Mit dieser Aufgabe beauftragte die Monarchin zuerst den Piaristen und vormaligen Schüler (bzw. Mitarbeiter) Ferdinand Kigler, ${ }^{23}$ der seine Erfahrungen bei der Leitung einer Normalschule in Wien und dann im Waisenhaus in Wartberg anwenden sollte. Dadurch entstand auch Idee, Felbigers Erfahrungen auch in Ungarn auszunutzen.

Am 23. Oktober 1778 wurde Felbiger zum Pressburger Domprobst ernannt. Schon kurz darauf (am 22. Januar 1779) tritt er sein Amt an. Die Ernennung in 
ein wichtiges Kirchenamt und zugleich die Erteilung vom ungarischen Indigenat brachten ihm eine entsprechende Versorgung und Akzeptanz im ungarischen Umfeld. ${ }^{24}$ Bald erlebte er jedoch sehr turbulente Zeiten, die mit einer Skepsis über die Neuorientierung der Schulreformen verbunden waren.

Die Probleme entstanden kurz nach Josephs II. Machtübernahme, als der neue Herrscher seine Interessen an intensiveren Aktivitäten im Volksschulwesen ankündigte. Bereits am Anfang seiner Regierung stimmte er der Ratio educationis zu und plädierte für ihre schnellere Implementierung..$^{25}$ Die Kontrolle der Einführung des ,verbesserten“ Unterrichts, d.h. des vorgeschriebenen Unterrichtsplans, der Lehrbücher, der Unterrichtsmethoden und der Finanzierung der Schulen und Lehrer, zeigte jedoch nur schwache Ergebnisse. ${ }^{26}$ Am Ende der 70er-Jahre gab es die ,verbesserten“ Schulen ausschließlich in größeren, meistens freien königlichen Städten. Die Schulen und Schüler erreichten zwar gute Resultate, ${ }^{27}$ sie hatten jedoch mit schweren materiellen Problemen zu kämpfen, die trotz der schriftlich bestätigten Verpflichtungen der Städte und Dörfer zur Finanzierung der Schulen entstanden. Es kam auch vor, dass der Unterricht manchmal überdimensioniert und für Schüler aus den niedrigeren Sozialschichten zu fordernd war. Es gab sogar Schulen, wo mehr Lehrer wirkten, als es notwendig gewesen wäre. Andererseits zeigten die Grundherren nur wenig Interesse an der Einführung der verbesserten Schulen auf ihren Herrschaften. Dies bestimmt auch aus dem Grund, dass die Existenz der Schulen stark von ihrer Unterstützung abhängig war. ${ }^{28}$

Abhilfe bei diesen Problemen sollte ein Wechsel am Posten des Pressburger Schulinspektors, des wichtigsten schulischen Postens für das gesamte Ungarn, schaffen. Anstelle von Caspar Pál ab Ehrenfels wurde Felbigers Kollege, Kanoniker Johann Schober, zum Schulinspektor ernannt. ${ }^{29}$ Zuerst sollte er innerhalb des Pressburger Komitats eine Untersuchung durchführen, um das Fortschreiten der Schulreformation zu dokumentieren und folglich die entsprechenden $\mathrm{Ma} \beta$ nahmen vorzuschlagen. Die Berichte der Assessoren beweisen, dass Dörfer und kleine Städte sowie die Bewohner selbst weder Interesse an den geforderten Änderungen noch Mittel zu deren Umsetzung hatten. ${ }^{30}$ Deswegen erschien Kigler - als eine im Schulwesen erfahrene und von den ungarischen Verhältnissen unabhängige Person - für diese Aufgaben als besonders geeignet. Die Beauftragung zur Kontrolle der Volksschulen innerhalb ganz Ungarns erteilte ihm am 14. November 1781 Joseph II. ${ }^{31}$ Dieser Schritt bedeutet zugleich auch den Anfang der allmählichen Unterordnung der ungarischen Schulverwaltungsorgane unter die Wiener Studienhofkommission. ${ }^{32}$ Felbiger wurde über die erwähnten Änderungen informiert und überhaupt nicht ignoriert. Seine Aufgabe wurde auch von Joseph II. persönlich näher bestimmt: Er beauftragte ihn mit der Anpassung seines Methodenbuches an die ungarischen Verhältnisse, um die Vereinheitlichung des Volkschulwesens innerhalb der ganzen Monarchie auf dieser Weise $\mathrm{zu}$ fördern. ${ }^{33}$ 
Der größte Einfluss Felbigers auf die Ausformung des Volkschulwesens in Ungarn ist dann in der Zeit um den Erlass des Toleranzpatents zu beobachten. ${ }^{34}$ Es waren auch die Protestanten, die über das Schicksal Felbigers in Ungarn - und zwar eher negativ - entschieden. Dazu ist es jedoch notwendig, ihre Stellungnahme zu den Schulreformen darzustellen, um ihre und Felbigers Positionen besser zu verstehen. Diese Zeit war trotz der im Toleranzpatent verankerten Erleichterungen in den Bedingungen für die Religionsausübung und Schulgründung recht kompliziert. Kurz vor seinem Erlass wurde das sog. Proiectum Budense angenommen, das verschiedene Maßnahmen und Unterrichtspläne brachte, die den einzelnen Schultypen angepasst wurden. Das Proiectum ergänzte die Ratio educationis und definierte die staatlichen Volksschulen als öffentliche Institutionen, die alle Bewohner ohne Ausnahmen finanziell unterstützen sollten. Das Problem lag darin, dass bis dato nur die katholischen Schulen vom Staat kontrolliert wurden. Die Forderung des Schulbesuchs, formuliert bereits in der Ratio und dann auch im Proiectum, bezog sich auch auf die nicht-katholischen Kinder, falls es vor Ort keine protestantische Schule gab - somit griff das Proiectum in die schulische Autonomie der Protestanten ein. Anderseits brachte auch das Toleranzpatent keine Lösung für den gemeinsamen Schulbesuch der Schüler verschiedener Konfessionen in Ortschaften, wo diese keine eigene Schule besaßen. Ebenfalls wurden keine entsprechenden Lehrbücher oder Befugnisse der Lehrer bestimmt. ${ }^{35}$ Die durch den Statthaltereirat organisierte Tagung, die all diese Probleme lösen sollte, begann am 1. März 1782 in Pressburg. Es war dies das erste offizielle Treffen der Repräsentanten der Staatsverwaltung und der katholischen Kirche mit den Repräsentanten der nicht-katholischen Denominationen in Ungarn. Felbiger und Kigler, damals beide mit der Reformierung der Volksschulen in Ungarn beauftragt, waren auch anwesend. Die katholische Seite und Schulorgane vertraten auch die Kanoniker - die Schulinspektoren Mandics, Erdélyi und Schober sowie der Präses dieser Kommission, Nicolaus Skerlec (Śkrlec). ${ }^{36}$ Die Lutheraner wurden durch Joseph Benczur, Gabriel Prónay, Samuel Tessedik und István Vay, die Reformierten durch István Hatványi und Gedeon Ráday, und die Orthodoxen nur von Theodor Janković vertreten.

Nicht einmal diese gemischte Kommission konnte den offenen Konflikt mit der Staatsverwaltung verhindern. Im Unterschied zur Vergangenheit hinderte die Protestanten nichts daran, sich mit ihren offiziellen Protesten gegen die Einschränkung ihrer Schulautonomie direkt an den Herrscher zu wenden. ${ }^{37}$ Obwohl den Protestanten bereits ausführliche Analysen über die Auswirkungen der Ratio educationis auf ihr Schulsystem zur Verfügung standen, verlangten sie aus taktischen Gründen die Tagung zu verschieben, damit sie sich angeblich besser vorbereiten und die Materialien gründlich auswerten könnten. ${ }^{38}$ Joseph II. bewilligte ihnen eine einjährige Pause, die dann zur Vorbereitung einer ausführlichen Publikation genutzt wurde. ${ }^{39}$ Darin wurden die Lehrbücher für die Elementarschulen 
und eigentlich das ganze System dieses Schultyps hart angegriffen. Dank den Kontakten der Protestanten mit Friedrich Nicolai und August Ludwig Schlözer gelang es ihnen, die Diskussion über die Organisation des Volksschulwesens und seine Qualität in die deutsche Presse zu bringen. ${ }^{40}$

Felbiger fühlte sich durch diese Kritik persönlich beleidigt und kurz danach reagierte er darauf mit seinem Werk Anekdoten zur Geschichte des Angriffes. ${ }^{41}$ Felbiger präsentierte darin die Stellungnahmen der ungarischen Protestanten und die steigende Kritik des ganzen Volksschulsystems, das vor nicht langer Zeit sowohl zuhause als auch im Ausland positiv bewertet worden war, in direktem Zusammenhang. Zu seiner Verteidigung brachte er ausführliche Informationen über die Grundsätze der russischen Schulreform, die damals unter der Leitung vom Staatsrat Aepinus und Theodor Janković begann. Er berichtete auch über die Grundsätze der Schulpolitik bezüglich der Nichtkatholiken, wie sie in der Ratio educationis definiert wurden und beschrieb den Verlauf der Tagung der gemischten Pressburger Kommission. In diesem Zusammenhang äußerte er sich, dass die einjährige Tagungsunterbrechung vor allem zur negativen Beeinflussung der öffentlichen Meinung im Ausland dienen sollte, was eigentlich auch die Anzeige der Edition von Angriff bereits am Anfang 1782(!) im Leipziger Bücher-Cata$\log$ beweise. ${ }^{42}$ Allem Anschein nach sah er in den Pressburger Lutheranern einen starken Gegner, weil er gerade ihnen die Autorenschaft von Angriff zuschrieb. Er vermutete sogar, dass der auf dem Titelblatt angegebene Druckort Stettin-Berlin fingiert und das Werk in Wirklichkeit in Pressburg erschienen sei.

Die Anekdoten konnten ihrem Autor jedoch nicht jenes Vertrauen zurückbringen, das er vor dem Konflikt mit den Protestanten genossen hatte. Die Zwistigkeiten betreffend der Aufgabe und Verwaltung des Volksschulwesens waren zu heftig dafür, dass sie ein Vertreter der gemäßigten katholischen Aufklärung zu lösen wusste..$^{43}$ Nach dem Ablauf der einjährigen Frist war klar, dass die Leitung des ganzen Schulwesens in die Hände von Gottfried van Swieten überging, der sich um seine Verstaatlichung bemüht habe. ${ }^{44}$ Van Swieten sah im Religionsunterricht nicht Ziel, sondern Mittel zur Erreichung des Staatszwecks - das Gemeinwohl für die Staatsuntertanen (Staatsbürger). Wäre der allgemeine Unterricht für alle gleich und würde der Schwerpunkt des Religionsunterrichts in ethischer Erziehung liegen, so sollte nichts den gemeinsamen Unterricht aller Kinder, ohne Unterschied ihrer Konfessionen hindern - nur der Katechismus solle separat vorgetragen werden. So entstand die Idee der vermischten Schulen, die jedoch über die Grenzen von Felbigers Toleranz hinausging. In diesem Punkt gelang die Felbiger's Aufgabe zu Ende und er sich aus der Schulverwaltung völlig entzog. Seine Aufgaben eines Domprobstres waren so wie so nicht einfach - er sollte sich an der Refomation der Kapitel beteiligen und widmete sich nur als Privatperson der Erziehung seiner Schützlinge, zwei kleinen Halbwaisen, Gebrüder Kofler... 
Meiner Meinung nach sollte man die Bewertung von Felbigers Verlegung nach Ungarn korrigieren und sie nicht als eine Erniedrigung betrachten, sondern eher als eine Erweiterung seiner Befugnisse auf einen anderen wesentlichen Teil der Habsburgermonarchie ansehen. Der spätere Verlust seiner dortigen Kompetenzen wurde eher durch die Änderung der allgemeinen Konzeption der Schulverwaltung in den Erbländern und später in der ganzen Monarchie verursacht als durch die bereits erwähnten Kontroversen mit dem Kaiser bezüglich der Erziehung der Soldatenkinder. Felbigers Schicksal ist auch mit Gottfried van Swieten verbunden, der während der Konzentration der Schulverwaltung in seinen Händen weder auf Felbigers Verdienste in Österreich noch in Ungarn achtete. ${ }^{45}$ Van Swieten bevorzugte in diesem Prozess natürlich solche Leute, die die Zentralisierungsund Toleranzidee vertraten und dabei gerade zu der Gruppe gehörten, die Felbiger aus dem Spiel brachte ...

\section{Quellen}

(Anon.): Freymuthige Beurtheilung der österreichischen Normalschulen und aller zum Behuf derselben gedruckten Schulschriften. Berlin - Stettin 1783.

Benda, Kálmán: Gerard und Gottfried van Swieten und die Schulreform in Ungarn. - In: Lesky, Erna; Wandruszka, Adam (Hg.): Gerard van Swieten und seine Zeit. Wien - Köln - Graz: Böhlau 1973, S. 164-174.

Csóka, Lajos: Der erste Zeitabschnitt staatlicher Organisierung des öffentlichen Unterrichtswesens in Ungarn (1760-1791). - In: Jahrbuch des Graf Klebelsberg Kuno Instituts für ungarische Geschichtsforschung in Wien, IX (1939), S. 45-124.

Engelbrecht, Helmut: Geschichte des österreichischen Bildungswesens, Bd. 3, Wien 1984.

Felbiger, Johann Ignaz von: Anekdoten zur Geschichte des Angriffes und der Vertheidigung der Normalschulen in den kaiserlich-königlichen Staaten. Frankfurt-Leipzig 1784.

Fináczy, Ernő: A magyarországi közoktatás története Mária Terézia korában, Bd. II, Budapest 1902.

Göckingk, L.F.G. von (Hg.): Reise des Herrn von Bretschneider nach London und Paris nebst Auszügen aus seinen Briefen an Herrn Friedrich Nicolai. Berlin - Stettin 1817.

Hüfner, Lorenz: Schlözer und Ungarn. Ein Beitrag zur Internationalen Wirksamkeit August Ludwig von Schlözers (Dissertation) Berlin (Ost) 1972.

(Anon.): INTOLERANZ des katholischen Klerus gegen die ungarischen Protestanten. O.O. 1792.

Kosáry, Domokos: Müvelödés a XVIII. századi Magyarországon. Budapest 1981.

Kovachich, Martin Georg (Hg.): Merkur von Ungarn oder Litterarzeitung für das Königreich Ungarn und dessen Kronländer, 1786, 1787, zugänglich online [Zutritt am 17.5.2018]: http://reader.digitale-sammlungen.de/en/fs1/object/display/bsb10733496_00009.html

Kowalská 1996: Kowalská, Eva: Dokumente aus dem Nachlass von Johann Ignaz von Felbiger. In: Mitteilungen des Österreichischen Staatsarchivs 44 (1996), S. 145-162.

Kowalská 1995: Kowalská, Eva: Johann Ignaz von Felbiger and the Hungarian monarchy. In: Studia historica slovaca 19 (1995), S. 73-94.

Kowalská 1993a: Kowalská, Eva: Kontroverzná tolerancia: Protestanti a školské reformy osvietenského obdobia. [Kontroverse Toleranz: Protestanten und Schulreformen der Aufklärung] In: Historické štúdie 34 (1993), S. 55-76. 
Kowalská 1993b: Kowalská, Eva: Zabudnutý pokus o reformu l’udových škôl. Kaktivite K. E. Fürstenberga voblasti l'udového školstva. [Der vergessene Versuch um Reformen der Volksschulen. Zur Aktivität von K. E. Fürstenberg in der Sphäre des Volkschulen] - In: Documenta Pragensia XI. (=Škola a město. Sborník př́íspěvků z konference „Škola a město“, konané ve dnech 5.-6.10.1992). Praha 1993, S. 135-139.

Kowalská 1991: Kowalská, Eva: Učebnice pre štátne l’udové školy na Slovensku koncom 18. storočia [Lehrbücher für staatliche Volkschulen in der Slowakei am Ende des 18. Jahrhunderts]. - In: Kniha 90, Matica slovenská 1991, S. 63-77.

Kowalská 1983: Kowalská, Eva: Bratislavské l’udové školstvo na konci 18. storočia [Pressburger Volksschulwesen am Ende des 18. Jahrhunderts] - In: Historický časopis 31 (1983), S. 776788.

Kunitsch, Michael: Biographien merkwürdiger Männer Österreichs, Bd. 3, Graz 1805.

Lambrecht 2006: Lambrecht, Karen: „Die nötige Erziehung in der Religion und in den bürgerlichen Pflichten “- die katholischen Reformbemühungen Johann Ignaz von Felbigers und Karl Egon von Fürstenbergs. - In: Bahlcke, Joachim (Hg.): Konfessionelle Pluralität als Herausforderung: Koexistenz und Konflikt in Spätmittelalter und Früher Neuzeit. Winfried Eberhard zum 65. Geburtstag. Leipzig, 2006, S. 575-601.

Lambrecht 2004: Karen: Tabelle und Toleranz. Johann Ignaz von Felbigers Reform der Volksschulbildung in Ostmitteleuropa. - In: Scheutz, Martin; Schmale, Wolfgang; Štefanová, Dana (Hg.): Orte des Wissens (= Jahrbuch der Österreichischen Gesellschaft zur Erforschung des achtzehnten Jahrhunderts) 18/19, (2004), S. 153-167.

Melton, James van Horn: Absolutism and the 18th-century origins of compulsory schooling in Prussia and Austria. Cambridge Univ. Press, 1988.

Nicolai, Friedrich: Beschreibung einer Reise durch Deutschland und die Schweiz im Jahre 1781, nebst Bemerkungen über Gelehrsamkeit, Industrie, Religion und Sitten. Bd. 4, Berlin 1784.

Parthey, Gustav C. F. (Hg.): Die Mitarbeiter an F. Nicolais Allgemeiner Deutscher Bibliothek nach ihren Namen und Zeichen, in zwei Register geordnet. Ein Beitrag zur deutschen Literaturgeschichte. Berlin 1842. Nachdruck Hildesheim 1973. zugänglich online [Zutritt am 17.5.2018]: Preßburger Zeitung, 1774, 1775, 1781, 1783, 1784, https:/www.difmoe.eu/d/periodical/uuid:1ec30e40-d452-11de-8dc5-000d606f5dc6

Schlözer 1783, 1786, 1789: Schlözer, August Ludwig von: Staats-Anzeigen, zugänglich online [Zutritt am 17.5.2018]: http://ds.ub.uni-bielefeld.de/viewer/toc/1944381/1/LOG_0000/

Wallaszky, Paulus: Conspectus rei publicae litterariae, 1. Ausgabe, Ofen 1785.

Wangermann, Ernst: Aufklärung und staatsbürgerliche Erziehung. Gottfried van Swieten als Reformator des österreichischen Unterrichtswesens 1781-1791. Wien: Verlag für Geschichte und Politik 1978.

\section{Anmerkungen}

1 (Anon.): Freymuthige Beurtheilung der österreichischen Normalschulen und aller zum Behuf derselben gedruckten Schulschriften. Berlin - Stettin 1783.

2 Die Studie entstand im Rahmen des Projekts APVV „Individual and Society - their Mutual Reflexion in Historical process".

3 Kosáry, Domokos: Müvelödés a XVIII. századi Magyarországon. Budapest 1981, S. 428, 455456, 461; Fináczy, Ernő: A magyarországi közoktatás története Mária Terézia korában, Bd. II, Budapest 1902, S. 7-54, 110-116; Engelbrecht, Helmut: Geschichte des österreichischen Bildungswesens, Bd. 3, Wien 1984. 
4 Melton, James van Horn: Absolutism and the 18th-century origins of compulsory schooling in Prussia and Austria. Cambridge: Cambridge Univ. Press 1988, S. 229-230.

5 Publiziert durch Kowalská, Eva: Dokumente aus dem Nachlass von Johann Ignaz von Felbiger. - In: Mitteilungen des Österreichischen Staatsarchivs 44 (1996), S. 145-162.

6 Um seine Aktivität in Ungarn zu entfalten, musste Felbiger zuerst das hiesige Indigenat erwerben. Maria Theresia bestätigte es feierlich am 23.10.1778, zusammen mit seiner Ernennung zum Probst.

7 Dazu Lambrecht, Karen: Tabelle und Toleranz. Johann Ignaz von Felbigers Reform der Volksschulbildung in Ostmitteleuropa. - In: Scheutz, Martin; Schmale, Wolfgang; Štefanová, Dana (Hg.): Orte des Wissens (= Jahrbuch der Österreichischen Gesellschaft zur Erforschung des achtzehnten Jahrhunderts) 18/19, (2004), S. 153-167.

8 Kowalská, Eva: Johann Ignaz von Felbiger and the Hungarian monarchy. - In: Studia historica slovaca 19 (1995), S. 73-94, hier 80-81.

9 Preßburger Zeitung, 21.12.1774, 11.1.1775.

10 Institutiones pro normalibus scholis sollten als Unterrichtsgegenstand in den Priesterseminaren eingeführt werden. Magyar Nemzeti Levéltár - Országos Levéltár (weiter MNL - OL): C 69, Collectio B. Normal. Resolutionum, A 593, fol. 28.

11 Über die Ersetzung des Canisius durch den „Wiener“ Katechismus, d. h. durch jenen, der an der Wiener Normalschule benutzt wurde, entschied man am 8.11.1774. Dagegen protestierten jedoch inbesondere die ungarischen Bischöfe heftig. Der erste Lehrer, der den neuen Katechismus benutzte, war der Lehrer an der Normalschule auf den kaiserlichen Gütern in Holitsch (Holíč). Nach dem Erlass der Verordnung am 27. 5. 1775 musste er jedoch auf diesen wieder verzichten. MNL - OL: C 69, A 593, fol. 217.

12 MNL - OL, C 69, Collectio B.Normal. Resolutionum, A 593, fol. 27.

13 Merkur von Ungarn, 1787, S. 773-774.

14 Merkur von Ungarn, 1787, S. 827-828, 830.

15 Den Text dieses Vorschlags publizierte: Merkur von Ungarn, 1787, S. 778-780.

16 Kowalská, Eva: Bratislavské l’udové školstvo na konci 18. storočia [Pressburger Volksschulwesen am Ende des 18. Jahrhunderts] - In: Historický časopis 31 (1983), S. 776-788, hier 779.

17 Kowalská, Eva: Učebnice pre štátne l’udové školy na Slovensku koncom 18. storočia [Lehrbücher für staatliche Volkschulen in der Slowakei am Ende des 18. Jahrhunderts]. - In: Kniha 90, Matica slovenská (1991), S. 63-77, hier 65.

18 Kowalská, Eva: Zabudnutý pokus o reformu l’udových škôl. K aktivite K. E. Fürstenberga voblasti l'udového školstva. [Der vergessene Versuch um Reformen der Volksschulen. Zur Aktivität von K. E. Fürstenberg in der Sphäre des Volkschulen]- In: Documenta Pragensia XI. (=Škola a město. Sborník příspěvků z konference „Škola a město“, konané ve dnech 5. - 6.10.1992). Praha 1993, S. 135-139; Lambrecht, Karen: „Die nötige Erziehung in der Religion und in den bürgerlichen Pflichten " - die katholischen Reformbemühungen Johann Ignaz von Felbigers und Karl Egon von Fürstenbergs. - In: Bahlcke, Joachim (Hg.): Konfessionelle Pluralität als Herausforderung: Koexistenz und Konflikt in Spätmittelalter und Früher Neuzeit. Winfried Eberhard zum 65. Geburtstag. Leipzig, 2006, S. 575-601.

19 Allgemeines Verwaltungsarchiv Wien, Studienhofkommission (weiter AVA Wien, SHK), 24 Schulbücher, kart. 109 Niederösterreich, fasc. 86a, II-III., fol. 11-13.

20 Kowalská: Učebnice, S. 65, 69.

21 Csóka, Lajos: Der erste Zeitabschnitt staatlicher Organisierung des öffentlichen Unterrichtswesens in Ungarn (1760-1791). - In: Jahrbuch des Graf Klebelsberg Kuno Instituts für ungarische Geschichtsforschung in Wien IX (1939), S. 45-124, hier 84-85.

22 Kowalská: Učebnice, S. 66-67. 
23 Nach dem Tod von Maria Theresia wurde Franz Balassa zum Rat der Ungarischen Hofkammer, dem Kronehüter und kroatischen Ban ernannt und engagierte sich fortan nicht mehr im Schulwesen. Pressburger Zeitung, 3.9.1783, 11.5. und 14.5.1785.

24 Kunitsch, Michael: Biographien merkwürdiger Männer Österreichs, Bd. 3, Graz 1805, S. 46-75.

25 Das Ernennungsdekret und die Bestätigung über die Erteilung des ungarischen Indigenats wurden am selben Tag, am 23.10.1778, herausgegeben. Slowakisches Nationalarchiv (im weiteren: SNA), Fonds Bratislavská kapitula, súkromný archív, C 30, fasc. 4, No. 13, bzw. ibidem, C 86, No. 23a.

26 Preßburger Zeitung, 12.5.1781. Als Unterlage für diese Nachricht diente das Intimat des Statthaltereirats vom 7.5.1781, worin dieser Schritt und des Kaisers weitere Pläne im Bereich des Schulwesens angemeldet wurden. Staatsarchiv Bratislava (im weiteren: SA Bratislava), Fonds ŽB I.-AC, 1781, fasc. 3, No. 15.

27 MNL - OL: C 69, 1784, Distr. Pos., fons 1, pos. 9, fol. 19-50 (Analyse aus dem Jahr 1781).

28 Rezensionen der Semestralprüfungen publizierte auch die Preßburger Zeitung (erster Bericht am 27.11.1776). Ähnlich auch Einladungen zu den öffentlichen Prüfungen, belegt für Pressburg, Tyrnau und Kaschau.

29 Den kanonischen Visitationen der Pfarreien im Pressburger und Nitraer Komitat, Anfang der 1780er-Jahre zufolge (insgesamt in mehr als 700 Dörfern und Kleinstädten) unterstützten die Grundherren die Schulen nur in 12 Lokalitäten. Ein ausführlicher Bericht über die Schulen im Neusohler (Banská Bystrica) Komitat wird in Országos Széchényi Könyvtár (weiter OSZK), in der Handschriftenabteilung, Acta Ürmeniana, Fol. Lat. 2996, fol. 38-40 aufbewahrt.

30 Ehrenfels wurde zum Kanoniker in Großwardein (Nagyvárad, heute Oradea, Rumänien) ernannt und verließ den Schuldienst. Schober wurde 1781, im Alter von 41 Jahren, Kanoniker.

31 SA Bratislava, Fonds ŽB I.-AC, 1781, fasc. 4, No. 74.

32 Kunitsch: Biographien, S. 50. Der Statthaltereirat informierte Franz Balassa mit einem Reskript am 29.11.1781 und die subordinierten Ämter mit einem Intimat am 7.12.1781.

33 Csóka: Der erste Zeitabschnitt, S. 93-96 spricht gar nicht über die mögliche Rolle von Felbiger in Ungarn und deutet nur seine Unterstützung seitens G. van Swietens an.

34 ,....alias vero peculiariter injunctum esset, ut praecaeteris Methodicus Libellus tandem pro Hungariae Regno, Partibusque eidem adnexis accomodetur, ac ita pro Cynosura, et Directione quorumvis Magistrorum publici Iuris reddatur; Quo ita bgne. intenta in docendo uniformitas, quae unico hocce fere medio sperari potest certius obtineri quaeat; Hucdum vero nil prorsus constaret quid, et in quantum ad effectum bgnarum. harum Dispositionem Nostrarum actium sit...Qua occasione id qq.F.Vris pro congruo Notitiae Statu clementer hisce significamus: quod posteaquam Negotium Scholarum Nationalium respectu Universarum Germanicarum Ditionum Nostrarum, hujatis unice Studiorum Commissionis Activitati, ac Manipulationi plene subjectissimus, constitutus ante hac Normalium Scholarum Director Praepositus Felbiger, munio ejusdem praemissa ratione cessante, ad repetendam stationem redeundum, quippe in Hungariae Regnum ad Praeposituram Suam, dirigendasque ibidem Normales, ac Nationales Scholas inviatus habeatur. dtto 7ae Xbris ,781. No8160. " OSZK, Handschriftenabteilung, Fol.lat. 104, fol. 589-590.

35 Bereits unter seiner Aufsicht verlief z.B. der Wettbewerb der Kandidaten für den Posten eines Volksschulinspektoren des Pressburger Schuldistrikts in der zweiten Hälfte des Jahres 1780. MNL - OL: C 69, 1780, Distr. Pos., fons 3, pos. 70, fol. 295-322.

36 Der königliche Rat und Universitätsbibliothekar Heinrich Bretschneider charakterisierte die Situation eindeutig: Man will nun gern die Protestanten hier unter das katholische, ungarische Studiensystem zwingen und das wäre schlimmer als Nontoleranz. Brief von H. Bretschneider an F. Nicolai, Ofen (Buda) am 8.4.1782. Edition durch Göckingk, L.F.G. von (Hg.): Reise des 
Herrn von Bretschneider nach London und Paris nebst Auszügen aus seinen Briefen an Herrn Friedrich Nicolai. Berlin - Stettin 1817, S. 291.

37 Die personelle Zusammensetzung der Kommission erwähnen Wallaszky, Paulus: Conspectus reipublicae litterariae, 1. Auflage, Ofen 1785, S. 325 und Felbiger, Johann Ignaz von: Anekdoten zur Geschichte des Angriffes und der Vertheidigung der Normalschulen in den kaiserlichköniglichen Staaten. Frankfurt - Leipzig 1784, S. 13-14 voneinander abweichend.

38 Noch während der Regierung von Maria Theresia verbat man den Nicht-Katholiken die kollektiv verfassten Petitionen der Herrscherin zu überreichen. Dazu (Anon.): INTOLERANZ des katholischen Klerus gegen die ungarischen Protestanten. O.O. 1792, S. 170.

39 Kowalská, Eva: Kontroverzná tolerancia: Protestanti a školské reformy osvietenského obdobia [Kontroverse Toleranz: Protestanten und Schulreformen der Aufklärungszeit]. - In: Historické štúdie 34 (1993), S.55-76, hier 57-58.

40 Das Werk von Parthey, G. C. F. (Hg.): Die Mitarbeiter an F. Nicolais Allgemeiner Deutscher Bibliothek nach ihren Namen und Zeichen, in zwei Register geordnet. Ein Beitrag zur deutschen Literaturgeschichte. Hildesheim 1973, erwähnt Bretschneider als einen Korrespondenten von Nicolais Allgemeine Deutsche Bibliothek. Bretschneider stand in reger Korrespondenz und auch im persönlichen Kontakt mit Nicolai, was sonst Bretschneiders Akzeptanz als Mitglied der Studienhofkommission seitens Gottfried van Swieten verhinderte. Nicolais Beurteilung des intellektuellen Niveaus der Wiener Gesellschaft in Nicolai, Friedrich: Beschreibung einer Reise durch Deutschland und die Schweiz im Jahre 1781, nebst Bemerkungen über Gelehrsamkeit, Industrie, Religion und Sitten. Bd. 4, Berlin 1784, 645-671 war jedoch gar nicht positiv (man kann dahinter Bretschneider vermuten). Die kontroversen Meinungen zum Konzept des Normalschulwesens veröffentlichte auch Ludwig August Schlözer in Bezug zu dessen Übernahme in Russland. Eine ähnliche Meinung äußerte er auch zu den vermischten Schulen in Ungarn. Siehe Staats-Anzeigen, 1783, Bd. 3, S. 257-278; ebenda 1786: Bd. 9, S. 408-421; ebenda 1789, Bd. 12, S. 463-468. Die Berichterstatter von Schlözer waren seine zahlreichen Studenten aus Ungarn; ihre Namen bringt Hüfner, Lorenz: Schlözer und Ungarn. Ein Beitrag zur Internationalen Wirksamkeit August Ludwig von Schlözers (Dissertation) Berlin (Ost) 1972. Zu den Kontakten mit der deutschen Pädagogik mehr bei Korabinszky, Johann Matthias: Privatnachricht an Menschenfreunde. Handschrift in OSZK, Quart. Germ. 869.

41 Den ganzen Titel siehe Anm. 35.

42 J. I. Felbiger, im Brief an F. Nicolai vom 16.1.1782, schreibt, dass er die Anzeige über das vorbereitete Werk zum ersten Mal am 13.1.1782 gesehen habe. Staatsbibliothek Preußischer Kulturbesitz Berlin, Handschr. Abt., F. Nicolai, Briefwechsel, No. 21.

43 Kowalská: Kontroverzná tolerancia, S. 60-65.

44 Wangermann, Ernst: Aufklärung und staatsbürgerliche Erziehung. Gottfried van Swieten als Reformator des österreichischen Unterrichtswesens 1781-1791. Wien: Verlag für Geschichte und Politik 1978, S. 44-50; Benda: Van Swieten, S. 169-170.

45 Engelbrecht, Geschichte, S. 118-119 erwähnt diese Möglichkeit nur am Rande. Auch Professor Mayer aus Wien erwähnte die Ernennung von Gottfried van Swieten zur wichtigsten Person im Schulwesen in seinem Brief an F. Nicolai am 11.12.1781 als eine Überraschung. Staatsbibliothek Preußischer Kulturbesitz, Berlin, Handschr. Abt., F. Nicolai, Briefwechsel, No. 48. 